\title{
Telangiectatic Patch on the Forehead
}

Katelyn Shea, MD; Joseph Pierson, MD; Deborah L. Cook, MD

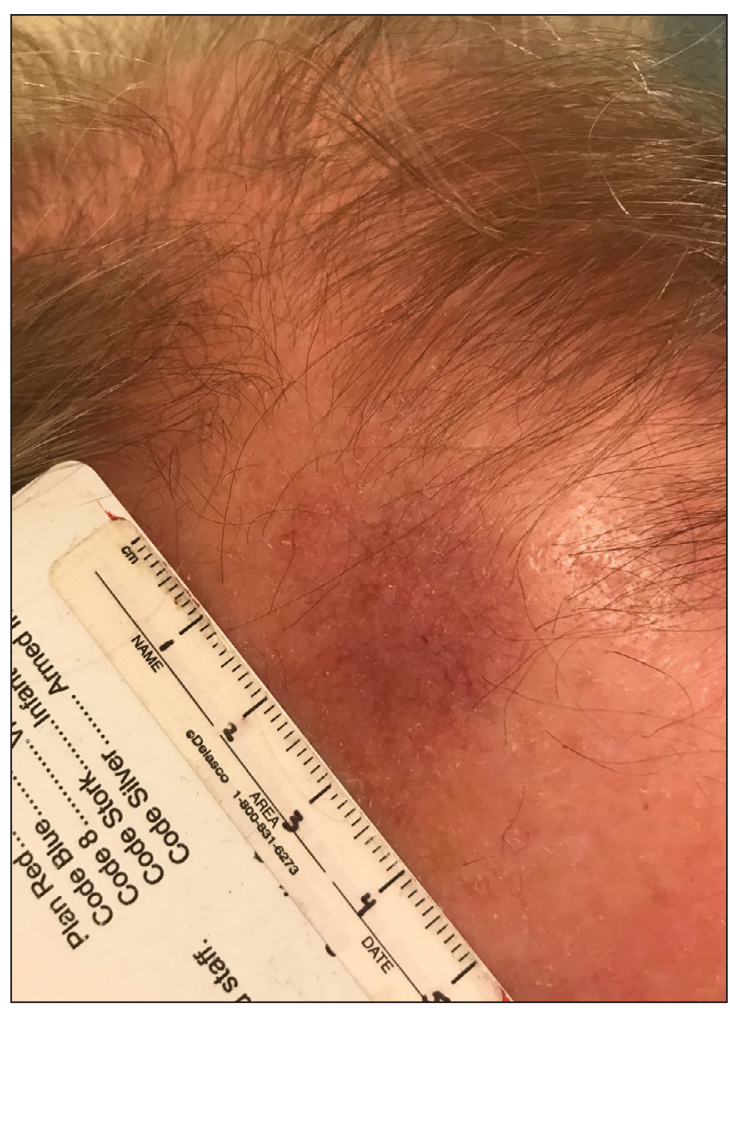

A 67-year-old man presented with a $2.5-\mathrm{cm}$, asymptomatic, plum-colored telangiectatic patch on the right side of the forehead of several months' duration. He had no similar skin findings elsewhere on the body and reported the patch had not changed. He did not recall any trauma to the area and denied prior surgical or radiation treatment. He had not yet tried any treatments for the lesion at the time of presentation. His medical history included insulin-dependent type 2 diabetes mellitus, hypertension, and obesity. He reported no fevers, chills, night sweats, or weight loss. A biopsy of the patch was performed.

\section{WHAT'S YOUR DIAGNOSIS?}
a. angiosarcoma
b. cutaneous B-cell lymphoma
c. Merkel cell carcinoma
d. pigmented purpuric dermatosis
e. rosacea

From the University of Vermont Medical Center, Burlington. Drs. Shea and Pierson are from the Division of Dermatology. Dr. Cook is from the Department of Pathology \& Laboratory Medicine.

The authors report no conflict of interest.

Correspondence: Katelyn Shea, MD, University of Vermont Medical Center, Division of Dermatology, 111 Colchester Ave, Burlington, VT 05465 (katelyn.shea@uvmhealth.org).

doi:10.12788/cutis.0141 


\section{THE DIAGNOSIS:}

\section{Cutaneous B-cell Lymphoma}

$H^{\mathrm{s}}$ istopathology was suggestive of cutaneous B-cell lymphoma (Figure). Further immunohistochemical studies including Bcl-6 positivity and Bcl-2 negativity in the large atypical cells supported a diagnosis of primary cutaneous follicle center lymphoma (PCFCL). The designation of primary cutaneous B-cell lymphoma includes several different types of lymphoma, including marginal zone lymphoma, diffuse large B-cell lymphoma, and intravascular lymphoma. To be considered a primary cutaneous lymphoma, there must be evidence of the lymphoma in the skin without concomitant evidence of systemic involvement, as determined through a full staging workup. Primary cutaneous follicle center lymphoma is an indolent lymphoma that most commonly presents as solitary or grouped, pink to plum-colored papules, plaques, nodules, and tumors on the scalp, forehead, or back. ${ }^{1}$ The lesions often are biopsied as suspected basal cell carcinomas or Merkel cell carcinomas (MCCs). Lesions on the face or scalp may easily evade diagnosis, as they initially may mimic rosacea or insect bites. Less common presentations include infiltrative lesions that cause rhinophymatous changes or scarring alopecia. Multifocal or disseminated lesions rarely can be observed. This case presentation is unique in its patchy appearance that clinically resembled angiosarcoma. ${ }^{2}$ When identified and treated, the disease-specific 5-year survival rate for PCFCL is greater than $95 \% .^{3}$

Merkel cell carcinoma was first described in 1972 and has been diagnosed with increasing frequency each year. ${ }^{4}$ It generally presents as an erythematous or violaceous, tender, indurated nodule on sun-exposed skin of the head or neck in elderly White men. However, other presentations have been reported, including papules, plaques, cystlike structures, pruritic tumors, pedunculated lesions, subcutaneous masses, and telangiectatic papules. ${ }^{5}$ Histopathologically, MCC is characterized by dermal nests and sheets of basaloid cells with finely granular salt and pepper-like chromatin. The histologic features can resemble other small blue cell tumors; therefore, the differential diagnosis can be broad. ${ }^{5}$ Immunohistochemistry that can confirm the diagnosis of MCC generally will be positive for cytokeratin 20 and neuroendocrine markers but negative for cytokeratin 7 and thyroid transcription factor 1. Merkel cell carcinoma is an aggressive tumor with a high risk for local recurrence and distant metastasis that carries a generally poor prognosis, especially when there is evidence of metastatic disease at presentation. 5,6

Rosacea can appear as telangiectatic patches, though generally not as one discrete patch limited to the forehead, as in our patient. Histologic features vary based on the age of the lesion and clinical variant. In early lesions there is a mild perivascular lymphoplasmacytic infiltrate within the dermis, while older lesions can have a mixed infiltrate crowded around vessels and adnexal structures. Granulomas often are seen near hair follicles and interspersed throughout the dermis with ectatic vessels and dermal edema. ${ }^{7}$
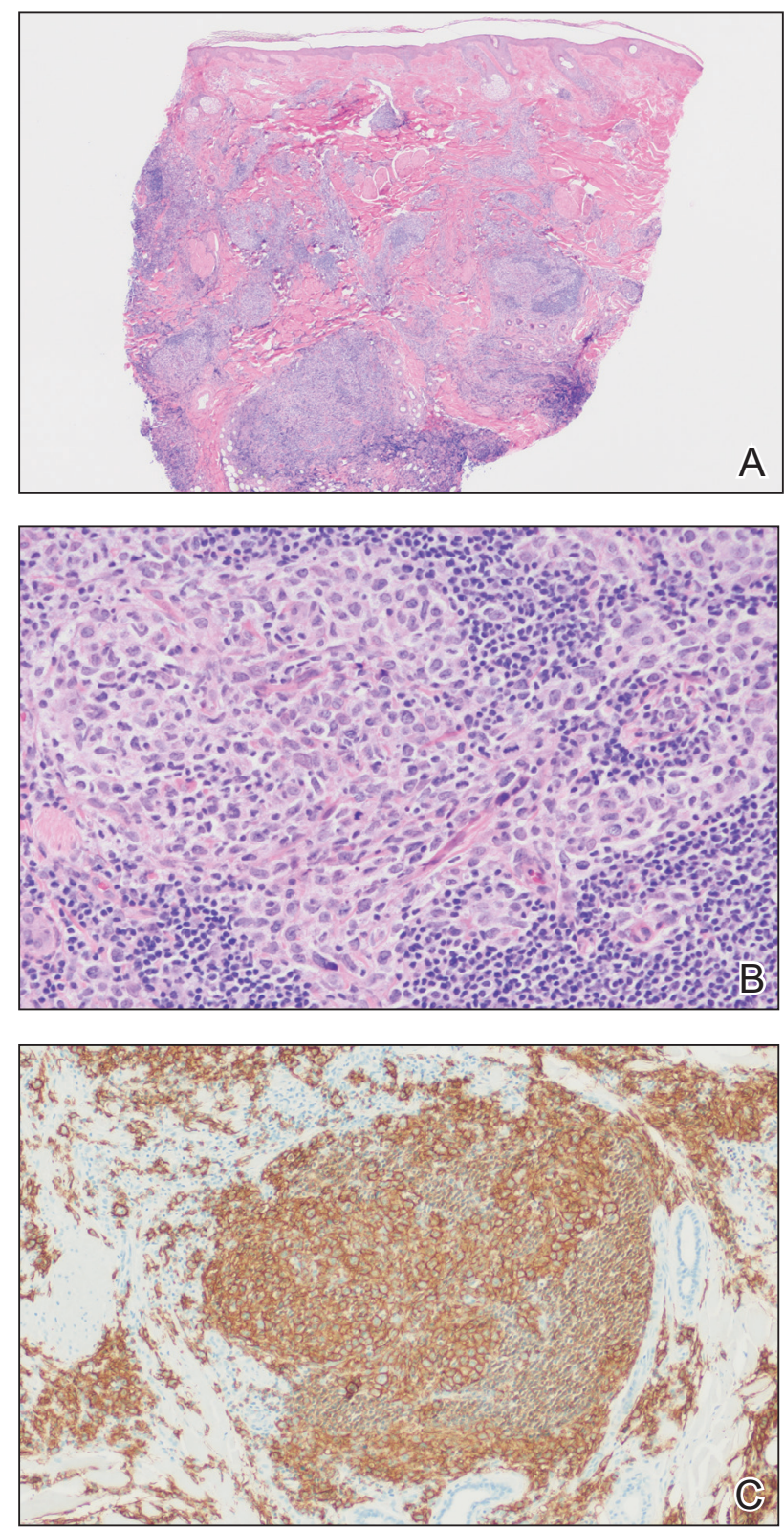

Cutaneous B-cell lymphoma. A, Histopathology showed an unremarkable epidermis with a dense nodular dermal infiltrate that extended to the subcutis $(H \& E$, original magnification $\times 20)$. B, Large pleomorphic cells with mitotic figures surrounded by a cuff of small uniform cells were seen (H\&E, original magnification $\times 200$ ). C, Staining of CD20 in large atypical cells was highlighted (original magnification $\times 100$ ). 
Angiosarcoma is divided into 3 clinicopathological subtypes: idiopathic angiosarcoma of the head and neck, angiosarcoma in the setting of lymphedema, and postirradiation angiosarcoma. ${ }^{7}$ Idiopathic angiosarcoma most closely mimics PCFCL, as it can present as single or multifocal nodules, plaques, or patches. Histologically, the 3 groups appear similar with poorly circumscribed, infiltrative, dermal tumors. The neoplastic endothelial cells have large hyperchromatic nuclei that protrude into vascular lumens. The prognosis for idiopathic angiosarcoma of the head and neck is poor, with a 5-year survival rate of $15 \%$ to $34 \%$, which often is due to delayed diagnosis. ${ }^{7}$

Pigmented purpuric dermatoses (PPDs) are chronic skin disorders characterized by purpura due to extravasation of blood from capillaries; the resulting hemosiderin deposition leads to pigmentation. ${ }^{7}$ There are various forms of PPD, which are classified into groups based on clinical appearance including Schamberg disease, purpura annularis telangiectodes of Majocchi, pigmented purpuric lichenoid dermatosis of Gougerot and Blum, lichen aureus, and others including eczematid and itching variants, which some consider to be distinct entities. Purpura annularis telangiectodes of Majocchi is the specific PPD that should be included in the clinical differential for PCFCL because it presents as annular patches with telangiectasias. Histologically, PPDs are characterized by a $\mathrm{CD} 4^{+}$lymphocytic infiltrate in the upper dermis with extravasated red blood cells and the presence of hemosiderin mostly within macrophages and a lack of true vasculitis. Clonality of the T cells has been shown, and there is some evidence that PPD may overlap with mycosis fungoides. However, this overlap mainly has been seen in patients with widespread lesions and would not apply to this case. In general, patients with PPD can be reassured of the benign process. In cases of widespread
PPD, patients should be followed clinically to assess for progression to mycosis fungoides, though the likelihood is low. ${ }^{7}$

Our patient underwent a full staging workup, which confirmed the diagnosis of PCFCL. He was treated with radiation to the forehead that resulted in clearance of the lesion. Approximately 2 years after the initial diagnosis, the patient was alive and well with no evidence of recurrence of PCFCL.

In conclusion, it is imperative to identify unusual, macular, vascular-appearing patches, especially on the head and neck in older individuals. Because the clinical presentations of PCFCL, angiosarcoma, rosacea, MCC, and PPD can overlap with one another as well as with other entities, it is necessary to have a high level of suspicion and low threshold to biopsy these types of lesions, as outcomes can be drastically different.

\section{REFERENCES}

1. Goyal A, LeBlanc RE, Carter JB. Cutaneous B-cell lymphoma. Hematol Oncol Clin North Am. 2019;33:149-161.

2. Massone C, Fink-Puches R, Cerroni L. Atypical clinical presentation of primary and secondary cutaneous follicle center lymphoma (FCL) on the head characterized by macular lesions. J Am Acad Dermatol. 2016;75:1000-1006.

3. Wilcox RA. Cutaneous B-cell lymphomas: 2016 update on diagnosis, risk-stratification, and management. Am J Hematol. 2016;91:1052-1055.

4. Conic RRZ, Ko J, Saridakis S, et al. Sentinel lymph node biopsy in Merkel cell carcinoma: predictors of sentinel lymph node positivity and association with overall survival. J Am Acad Dermatol. 2019;81:364-372

5. Coggshall K, Tello TL, North JP, et al. Merkel cell carcinoma: an update and review: pathogenesis, diagnosis, and staging. J Am Acad Dermatol. 2018;78:433-442.

6. Tello TL, Coggshall K, Yom SS, et al. Merkel cell carcinoma: an update and review: current and future therapy. J Am Acad Dermatol. 2018;78:445-454.

7. Patterson JW, Hosler GA. Weedon's Skin Pathology. 4th ed. China: Churchill Livingstone Elsevier; 2016. 